\title{
Numerical Study of Droplet Generation in Co-flow Microfluidics
}

\author{
Zhe (Ashley) Chen ${ }^{1}$, Fenglei Huang ${ }^{1,2}$, Peichun Amy Tsai ${ }^{1}$, Alexandra Komrakova ${ }^{1}$ \\ ${ }^{1}$ Department of Mechanical Engineering, University of Alberta, Edmonton, Canada \\ ${ }^{2}$ School of Chemical Engineering, Beijing University of Chemical Technology, Beijing, China \\ *zhe8@ualberta.ca
}

\begin{abstract}
Droplet-based microfluidics has recently become an emerging platform for generating useful and controllable monodisperse droplets. Consequently, microfluidic droplet devices have a variety of beneficial applications in the fields of chemical engineering, analytical chemistry, pharmaceutical, material engineering, and lab-on-a-chip technologies. During the formation process of microfluidic droplets, surfactants are often added experimentally to aid the stability of emulsions. However, additive surfactants can significantly influence the droplet dynamics because their spatially-varying concentrations can alter the interfacial tension locally, thereby inducing a Marangoni stress at the liquid-liquid interfaces and affecting the flow field. In this work, we numerically investigate the flow dynamics and emulsion size of surfactant-laden droplets in a co-flow microfluidic device for monodisperse droplet generation. As a benchmark case, we first simulate and validate the droplet generation process in a free-surfactant environment. More specifically, we perform two-dimensional numerical simulations of an immiscible liquid-liquid co-flow, with equal density and viscosity of the liquids in microchannels. A diffuse interface phase-field lattice Boltzmann method (LBM) is used to calculate the flow field. By altering the flow rate of the dispersed and continuous phases, we observed the monodisperse droplets with varying sizes. We investigate the effect of the flow-rate ratio on the size of monodisperse droplets generated in the coflow microfluidics. Consistent with recent experimental results, we found both squeezing and dripping mode of the drop generation in the Capillary number range between $4 \cdot 10^{-3}$ and $8 \cdot 10^{-2}$. In addition, we study the influences of Reynolds number and Capillary number on both the dynamics of droplet generation and the final size of the emulsions. Our near-future work includes the simulations using immiscible liquids with large density and viscosity ratio. We will further discuss our simulation results of the surfactant effect on the emulsion dynamics and size in co-flow microfluidics.
\end{abstract}

